\title{
Sweeping Surface of Center Curve on Surface in Euclidean 3-space $\mathbf{E}^{3}$
}

\author{
${ }^{1}$ RASHAD A. ABDEL-BAKY, ${ }^{2}$ FATEMAH MOFARREH \\ ${ }^{1}$ Department of Mathematics; Faculty of Science, University of Assiut, Assiut 71516; \\ EGYPT \\ ${ }^{2}$ Mathematical ScienceDepartment; Faculty of Science; \\ Princess Nourah bint Abdulrahman University, Riyadh 11546, SAUDI ARABIA
}

\begin{abstract}
For the curve on the regular surface, there is moving frame with this thatis named Darboux frame. Sweeping surfaces through the curve associated with Darboux frame are introduced and their geometrical properties are investigated. Moreover, we obtain the necessary and sufficient conditions of this kind of surfaces to be developable ruled surfaces. Finally, an example to illustrate the application of the results is introduced.
\end{abstract}

Key-Words: Darboux frame, Sweeping surface, Singularity.

Received: January 8, 2021. Revised: April 20, 2021. Accepted: May 10, 2021. Published: May 30, 2021.

\section{Introduction}

Sweeping surfaces are generated by the motion of the plane curve ( profile curve or generatrix) while the movement of the plane in the space has the same direction of the normal for the plane. In geometrical modeling, sweeping is an essential and useful tool and it has some applications in networks of blood vessels, neurons in medicine and hose systems in industrial environments. The idea depends on choosing a geometrical object, that is called generator, and sweeping it along a spine curve, which is called trajectory [1-13].

Several studies in the previous literature that may be inclusived in the area of our treatise are as follows: Ylldiz et al. [2] conducted the geometrical observation of the ascending colons for several domestic animals as pigs, horses, ruminants, and dogs; these ascending colons were shown to have the tubular shape along the special curve, which is the application for tubular surfaces in medicine. Blaga [3] applied tubular surfaces as swept surfaces and put forward new approaches for the realization for tubular and canal surfaces in addition to showing applications of tubular surfaces in scientific visualization. Karacan and Yayll [5] studied geodesics of tubular surfaces at Minkowski 3-space. Karacan et al. [7] investigated the general and linear Weingarten conditions of tubular surfaces by the use of the Gaus- sian and mean curvature of the surface in Euclidean 3-space. Doğan [8] intended generalized canal surfaces and some special curves on them in his dissertation. Recently, tubular and canal surfaces have been also represented as quaternionic by several authors $[9,10]$.

In view of the mentioned references, tubular surface, string, pipe surface, and canal surface are considered as different names for the sweeping surfaces. So far as we know, there is no previous studies in regard to curves that lying at surfaces as the initial objects with the consideration of [13, 14], this study focuses on the geometrical properties of sweeping surfaces whose center curves in surfaces at Euclidean 3 -space $E^{3}$. Furthermore, in kinematics, the sweeping surfaces, the ruled surfaces, are introduced as one-dimensional line manifolds created by oriented moving line in the space, playing an important role of the line trajectory. As a consequence, considering the sweeping surfaces as a special ruled surfaces is important in both kinematics and differential line geometry theory.

In this work, the differential geometry of the sweeping surface with Darboux frame is developed. We also show that the parametric curves on this surface are lines of curvature. Then we study local singularities and convexity of a sweeping surface. In terms of this, we derived the necessary and sufficient condition of the sweeping 
surface to become the developable ruled surface. Additionally, an example of application is introduced and explained in detail.

\section{Preliminaries}

Suppose $\alpha: I \subseteq R \rightarrow E^{3}$ is the unit speed curve, $\kappa(s)$ and $\tau(s)$ define natural curvature and torsion of $\alpha=\alpha(s)$, in the same order. Suppose $\alpha^{\prime \prime}(s) \neq 0$ for any $s \in[0, L]$, which gives the straight line. At this work, $\alpha^{\prime}(s)$ defines derivative of $\alpha$ respecting to the arc length parameter $s$. For all points of $\alpha(s)$, this set of $\{\mathbf{t}(s)$, $\left.\mathbf{n}(s), \mathbf{e}_{1}(s)\right\}$ is named the Serret-Frenet frame through $\alpha(s)$, where $\mathbf{t}(s)=\alpha^{\prime}(s)$ is a unit tangent, $\mathbf{n}(s)=\alpha^{\prime \prime}(s) /\left\|\alpha^{\prime \prime}(s)\right\|$ is a unit principal normal, and $\mathbf{b}(s)=\mathbf{t}(s) \times \mathbf{n}(s)$ is a unit binormal vector. The arc-length derivative of the SerretFrenet frame is given as:

$$
\left(\begin{array}{l}
\mathbf{t}^{\prime} \\
\mathbf{n}^{\prime} \\
\mathbf{b}^{\prime}
\end{array}\right)=\left(\begin{array}{lll}
0 & \kappa(s) & 0 \\
-\kappa(s) & 0 & \tau(s) \\
0 & -\tau(s) & 0
\end{array}\right)\left(\begin{array}{l}
\mathbf{t} \\
\mathbf{n} \\
\mathbf{b}
\end{array}\right) .
$$

Consider $F$ is the regular surface, and $\alpha: I \subseteq$ $R \rightarrow F$ is the unit speed curve at $F$. On this surface, the Darboux frame is $\left\{\alpha(s) ; \mathbf{e}_{1}, \mathbf{e}_{2}, \mathbf{e}_{3}\right\}$; $\mathbf{e}_{1}(s)$ is a unit tangent vector to $\alpha(s), \mathbf{e}_{3}=\mathbf{e}_{3}(s)$ is a surface unit normal restricted to $\alpha$, and $\mathbf{e}_{2}(s)=\mathbf{e}_{3} \times \mathbf{e}_{1}$ is a unit tangent to this surface $F$. Then, the rotation matrix between Serret-Frenet frame and Darboux frame is

$$
\left(\begin{array}{l}
\mathbf{t} \\
\mathbf{n} \\
\mathbf{b}
\end{array}\right)=\left(\begin{array}{lll}
1 & 0 & 0 \\
0 & \cos \theta & \sin \theta \\
0 & -\sin \theta & \cos \theta
\end{array}\right)\left(\begin{array}{l}
\mathbf{e}_{1} \\
\mathbf{e}_{2} \\
\mathbf{e}_{3}
\end{array}\right) .
$$

The variation of the Darboux frame through the curve $\alpha(s)$ is described using next equations:

$$
\left(\begin{array}{l}
\mathbf{e}_{1}^{\prime} \\
\mathbf{e}_{2}^{\prime} \\
\mathbf{e}_{3}^{\prime}
\end{array}\right)=\left(\begin{array}{lll}
0 & \kappa_{g} & \kappa_{n} \\
-\kappa_{g} & 0 & \tau_{g} \\
-\kappa_{n} & -\tau_{g} & 0
\end{array}\right)\left(\begin{array}{l}
\mathbf{e}_{1} \\
\mathbf{e}_{2} \\
\mathbf{e}_{3}
\end{array}\right),
$$

where

$$
\left.\begin{array}{l}
\kappa_{n}(s)=\kappa \sin \theta=<\alpha^{\prime \prime}, \mathbf{e}_{3}>, \\
\kappa_{g}(s)=\kappa \cos \theta=\operatorname{det}\left(\alpha^{\prime}, \alpha^{\prime \prime}, \mathbf{e}_{3}\right), \\
\tau_{g}(s)=\tau-\theta^{\prime}=\operatorname{det}\left(\alpha^{\prime}, \mathbf{e}_{3}, \mathbf{e}_{3}^{\prime}\right) .
\end{array}\right\}
$$

We call $\kappa_{g}=\kappa_{g}(s)$ a geodesic curvature, $\kappa_{n}=$ $\kappa_{n}(s)$ the normal curvature, and $\tau_{g}=\tau+\theta^{\prime}$ the geodesic torsion of $\alpha(s)$, in the same order. The geodesics, line of curvatures, and asymptotic lines on a smooth surface as quantities can be characterized, as loci along which $\kappa_{g}=0, \tau_{g}=0$, and $\kappa_{n}=0$, in the same order [1-3].

\section{$3 \quad$ Sweeping surfaces with Darboux frame}

In this section, we shall give the parametric representation of sweeping surfaces through a spine curve $\alpha(s)$ of the surface $F$ in the following: The sweeping surface associated to $\alpha(s)$, is the envelope of the one-parameter family of unit spheres, with the center on the curve $\alpha(s)$. Clearly, the contact between the spheres from the family and the sweeping surface is the great circle of the unit sphere, lying on the subspace $S p\left\{\mathbf{e}_{2}, \mathbf{e}_{3}\right\}$, of the spine curve $\alpha(s)$. Let us describe, then, a very simple method of representation of the sweeping surface. Take the parameter along $\alpha(s)$ to be one of the parameters and define by $\mathbf{Q}$ the position vector joining the point from the curve $\alpha(s)$ with the other from the sweeping surface. Therefore, it is clear that

$$
M: \mathbf{Q}=\alpha(s)+\mathbf{x},
$$

where the unit vector $\mathbf{x}$ itself lies in the subspace $S p\left\{\mathbf{e}_{2}, \mathbf{e}_{3}\right\}$. Let us denote by the angle $\vartheta$ between the vectors $\mathbf{x}$ and $\mathbf{e}_{2}$. Therefore,

$$
\mathbf{x}(\vartheta)=\cos \vartheta \mathbf{e}_{2}+\sin \vartheta \mathbf{e}_{3},
$$

which is the characteristic circle of sweeping surface. Combining Eqs. (5) and (6), we see that we obtained a parameterization of the sweeping surface,

$$
M: \mathbf{Q}(s, \vartheta)=\alpha(s)+\cos \vartheta \mathbf{e}_{2}(s)+\sin \vartheta \mathbf{e}_{3}(s) .
$$

Parametrizing $M$ in this way excludes sweeping surfaces with stationary vector $\mathbf{e}_{1}$, whose geometrical properties have less interest and easier to be explored.

Remark 3.1. Clearly, if $\alpha(s)$ defines the straight line, then the sweeping surface associated to it is just the circular cylinder, having $\alpha(s)$ as symmetry axis. If, on the other hand, $\alpha(s)$ is a circle, then the corresponding sweeping surface is a torus.

\subsection{Geometrical properties}

Using Eq. (3), leads to

$$
\left.\begin{array}{rl}
\mathbf{Q}_{\vartheta}(s, \vartheta)= & -\sin \vartheta \mathbf{e}_{2}+\cos \vartheta \mathbf{e}_{3}, \\
\mathbf{Q}_{s}(s, \vartheta)= & \left(1-\kappa_{g} \cos \vartheta-\kappa_{n} \sin \vartheta\right) \mathbf{e}_{1} \\
& +\tau_{g} \mathbf{Q}_{\vartheta}(s, \vartheta),
\end{array}\right\}
$$

and

$$
\mathbf{N}(s, \vartheta):=\frac{\mathbf{Q}_{\vartheta} \times \mathbf{Q}_{s}}{\left\|\mathbf{Q}_{\vartheta} \times \mathbf{Q}_{s}\right\|}= \pm\left(\cos \vartheta \mathbf{e}_{2}+\sin \vartheta \mathbf{e}_{3}\right) .
$$


The sign is depending on the sign of $\left(1-\kappa_{g} \cos \vartheta-\right.$ $\left.\kappa_{n} \sin \vartheta\right)$ Eq. (9) shows that the surface normal $\mathbf{N}(s, \vartheta)$ is contained in the the subspace $S p\left\{\mathbf{e}_{2}\right.$, $\left.\mathbf{e}_{3}\right\}$, because it is perpendicular to $\mathbf{e}_{1}$. From Eqs. (8), it is easily checked that the coefficients of the first fundamental form $g_{11}=<\mathbf{Q}_{\mathbf{s}}, \mathbf{Q}_{\mathbf{s}}>, g_{12}=<$ $\mathbf{Q}_{s}, \mathbf{Q}_{\vartheta}>$ and $g_{22}=<\mathbf{Q}_{\vartheta}, \mathbf{Q}_{\vartheta}>$ are given by

$$
\begin{gathered}
g_{11}=\left(1-\kappa_{g} \cos \vartheta-\kappa_{n} \sin \vartheta\right)^{2}+\tau_{g}^{2} \\
, g_{12}=\tau_{g}, \quad g_{22}=1 .
\end{gathered}
$$

To compute the second fundamental form of $M$, we have to calculate the following

$$
\begin{aligned}
& \mathbf{Q}_{s s}=\left[-\left(\kappa_{g}^{\prime} \cos \vartheta+\kappa_{n}^{\prime} \sin \vartheta\right)\right. \\
& \left.+\tau_{g}\left(\kappa_{g} \sin \vartheta-\kappa_{n} \cos \vartheta\right)\right] \mathbf{e}_{1} \\
& +\left[\kappa_{g}\left(1-\kappa_{n} \sin \vartheta\right)-\left(\kappa_{g}^{2}+\tau_{g}^{2}\right) \cos \vartheta\right. \\
& \left.-\tau_{g}^{\prime} \sin \vartheta\right] \mathbf{e}_{2} \\
& +\left[\kappa_{n}\left(1-\kappa_{g} \cos \vartheta\right)-\left(\kappa_{g}^{2}+\tau_{g}^{2}\right) \sin \vartheta\right. \\
& \left.+\tau_{g}^{\prime} \cos \vartheta\right] \mathbf{e}_{3}, \\
& \mathbf{Q}_{s \vartheta}=\left(\kappa_{g} \sin \vartheta-\kappa_{n} \cos \vartheta\right) \mathbf{e}_{1} \\
& -\tau\left(\cos \vartheta \mathbf{e}_{2}+\sin \vartheta \mathbf{e}_{3}\right), \\
& \mathbf{Q}_{\vartheta \vartheta}=-\cos \vartheta \mathbf{e}_{2}-\sin \vartheta \mathbf{e}_{3} .
\end{aligned}
$$

It gives the elements for second fundamental form $h_{11}=<\mathbf{Q}_{s s}, \mathbf{N}>, h_{12}=<\mathbf{Q}_{s \vartheta}, \mathbf{N}>$, and $h_{22}=<\mathbf{Q}_{\vartheta \vartheta}, \mathbf{N}>$ are given by

$$
\left.\begin{array}{c}
h_{11}=\left(1-\kappa_{g} \cos \vartheta-\kappa_{n} \sin \vartheta\right)\left(\kappa_{g} \cos \vartheta\right. \\
\left.\quad+\kappa_{n} \sin \vartheta\right)+\tau_{g}^{2}, \\
h_{12}=\tau_{g}, h_{22}=-1 .
\end{array}\right\}
$$

The Gaussian and mean curvature of the sweeping surface around the regular point are obtained, respectively, as

$K(s, \vartheta):=\frac{h_{11} h_{22}-h_{12}^{2}}{g_{11} g_{22}-g_{12}^{2}}=\frac{\kappa_{g} \cos \vartheta+\kappa_{n} \sin \vartheta}{1-\kappa_{g} \cos \vartheta-\kappa_{n} \sin \vartheta}$,

and

$$
\begin{array}{r}
H(s, \vartheta):=\frac{g_{22} h_{11}-2 g_{12} h_{12}+g_{11} h_{22}}{2\left(g_{11} g_{22}-g_{12}^{2}\right)} \\
=\frac{2\left(\kappa_{g} \cos \vartheta+\kappa_{n} \sin \vartheta\right)-1}{2\left(1-\kappa_{g} \cos \vartheta-\kappa_{n} \sin \vartheta-1\right)} .
\end{array}
$$

Proposition 3.1. For the sweeping surface $M$ represented by Eq. (7), the values of $K(s, \vartheta)$, and $H(s, \vartheta)$ are independent of the geodesic torsion for the spine curve $\alpha(s)$.

Proposition 3.2. Suppose $\alpha: I \subseteq R \rightarrow F$ is the unit speed curve on a surface $F$, then we state the following:

(1) If $\alpha$ is a geodesic on $F$, then the Gaussian and mean curvature of the sweeping surface $M$ are:

$$
K(s, \vartheta)=\frac{\kappa_{n} \sin \vartheta}{1-\kappa_{n} \sin \vartheta}
$$

and

$$
H(s, \vartheta)=\frac{2 \kappa_{n} \sin \vartheta-1}{2\left(1-\kappa_{n} \sin \vartheta-1\right)} .
$$

(2) If $\alpha$ is an asymptotic on $F$, then the Gaussian and mean curvature of the sweeping surface $M$ are

$$
K(s, \vartheta)=\frac{\kappa_{g} \cos \vartheta}{1-\kappa_{g} \cos \vartheta},
$$

and

$$
H(s, \vartheta)=\frac{2 \kappa_{g} \cos \vartheta-1}{2\left(1-\kappa_{g} \cos \vartheta-1\right)} .
$$

On the other hand, from Eq. (7) it is easily checked that the isoparametric curve

$\zeta(\vartheta):=\mathbf{Q}\left(\vartheta, s_{0}\right)=\alpha\left(s_{0}\right)+\cos \vartheta \mathbf{e}_{2}\left(s_{0}\right)+\sin \vartheta \mathbf{e}_{3}\left(s_{0}\right)$,

is the planar unit speed curve. The unit tangent vector to $\zeta(\vartheta)$ is

$$
\mathbf{T}_{\zeta}(\vartheta)=-\sin \vartheta \mathbf{e}_{2}\left(s_{0}\right)+\cos \vartheta \mathbf{e}_{3}\left(s_{0}\right),
$$

therefore the unit principal normal vector of $\zeta(\vartheta)$ is written as

$\mathbf{N}_{\zeta}=\mathbf{e}_{1}\left(s_{0}\right) \times \mathbf{T}_{\zeta}(u)=\cos \vartheta \mathbf{e}_{2}+\sin \vartheta \mathbf{e}_{3}=\mathbf{N}\left(s_{0}, \vartheta\right)$.

Hence, the surface normal $\mathbf{N}\left(s_{0}, \vartheta\right)$ is parallel to the principal normal $\mathbf{N}_{\zeta}$, which means the curve $\zeta(\vartheta)$ is the geodesic, and cannot be asymptotic curve on $M$.

Proposition 3.3. Suppose $\alpha: I \subseteq R \rightarrow F$ is the unit speed curve at a surface $F$. Then the $s$-parameter curves are asymptotic curves on $M$ if and only if

$$
\vartheta=\tan ^{-1}\left(\frac{\kappa_{n} \pm \kappa_{g} \sqrt{\kappa_{n}^{2}+\kappa_{g}^{2}-\sigma^{2}}}{\kappa_{g} \pm \kappa_{n} \sqrt{\kappa_{n}^{2}+\kappa_{g}^{2}-\sigma^{2}}}\right)
$$

where

$$
\sigma(s)=\frac{1}{2}\left[1 \pm \sqrt{1-4 \tau_{g}^{4}}\right] .
$$

Proof. The s- parameter curves are asymptotic 
curves on $M$ if and only if

$$
\left\langle\mathbf{N}, \mathbf{Q}_{s s}\right\rangle=0 \Leftrightarrow
$$

$\left(\kappa_{g} \cos \vartheta+\kappa_{n} \sin \vartheta\right)^{2}-\left(\kappa_{g} \cos \vartheta+\kappa_{n} \sin \vartheta\right)-\tau_{g}^{2}$ $=0$.

It follows that

$$
\begin{gathered}
\sin \vartheta=\frac{\kappa_{n} \pm \kappa_{g} \sqrt{\kappa_{n}^{2}+\kappa_{g}^{2}-\sigma^{2}}}{\kappa_{n}^{2}+\kappa_{g}^{2}}, \text { and } \\
\cos \vartheta=\frac{\kappa_{g} \pm \kappa_{n} \sqrt{\kappa_{n}^{2}+\kappa_{g}^{2}-\sigma^{2}}}{\kappa_{n}^{2}+\kappa_{g}^{2}}
\end{gathered}
$$

we therefore obtain Eq. (15) .

\subsubsection{Singularity and lines of curvature}

Singularities and lines of curvature are important to understand properties of sweeping surfaces and they will be studied as coming: The sweeping surface $M$ has singular points if and only if the first derivatives are linearly dependent, that is,

$$
\mathbf{Q}_{\vartheta} \times \mathbf{Q}_{s}=\left(1-\kappa_{g} \cos \vartheta-\kappa_{n} \sin \vartheta\right) \mathbf{N}=\mathbf{0} .
$$

Since $\mathbf{N}$ is not zero unit vector, therefore

$$
1-\kappa_{g} \cos \vartheta-\kappa_{n} \sin \vartheta=0,
$$

that is,

$$
\begin{gathered}
\sin \vartheta=\frac{\kappa_{n} \pm \kappa_{g} \sqrt{\kappa_{n}^{2}+\kappa_{g}^{2}-1}}{\kappa_{n}^{2}+\kappa_{g}^{2}} \text {, and } \\
\cos \vartheta=\frac{\kappa_{g} \pm \kappa_{n} \sqrt{\kappa_{n}^{2}+\kappa_{g}^{2}-1}}{\kappa_{n}^{2}+\kappa_{g}^{2}} .
\end{gathered}
$$

Thus, two singular points occur on every generating circle. By the connect of these two sets of singular points we have two curves that include all singular points of the sweeping surface. Using Eq. (7) gives the expression of these two curves as

$$
\begin{gathered}
\gamma(s)=\alpha(s)+\frac{\kappa_{g} \pm \kappa_{n} \sqrt{\kappa_{n}^{2}+\kappa_{g}^{2}-1}}{\kappa_{n}^{2}+\kappa_{g}^{2}} \mathbf{e}_{2} \\
+\frac{\kappa_{n} \pm \kappa_{g} \sqrt{\kappa_{n}^{2}+\kappa_{g}^{2}-1}}{\kappa_{n}^{2}+\kappa_{g}^{2}} \mathbf{e}_{3} .
\end{gathered}
$$
lary:

The previous analysis leads to the next corol-

Corollary 3.1. The sweeping surface $M$ represented by Eq. (7), has no singular points if the condition

$$
1-\kappa_{g} \frac{\kappa_{g} \pm \kappa_{n} \sqrt{\kappa_{n}^{2}+\kappa_{g}^{2}-1}}{\kappa_{n}^{2}+\kappa_{g}^{2}}-\kappa_{n} \frac{\kappa_{n} \pm \kappa_{g} \sqrt{\kappa_{n}^{2}+\kappa_{g}^{2}-1}}{\kappa_{n}^{2}+\kappa_{g}^{2}} \neq 0,
$$

is satisfied

According to theory of line of curvature, if every generating circle is lines of curvature, consequently $\mathbf{N}_{\vartheta}=\lambda(\vartheta) \mathbf{Q}_{\vartheta}$, where $\lambda(\vartheta)$ is a differentiable function of $\vartheta$. Using algebraic manipulations, it is founded that the generating circles are lines of curvature if and only if

$$
\tau_{g}\left(1-\kappa_{g} \cos \vartheta-\kappa_{n} \sin \vartheta\right)=0 .
$$

for any $s$, and $\vartheta$. Clearly, there is two cases, and they are as coming:

Case (1) occurs if $\tau_{g} \neq 0$, and $1-\kappa_{g} \cos \vartheta-$ $\kappa_{n} \sin \vartheta=0$. Therefore there are two singular points at the generating circle, they are located at

$$
\vartheta=\tan ^{-1}\left(\frac{\kappa_{n} \pm \kappa_{g} \sqrt{\kappa_{n}^{2}+\kappa_{g}^{2}-1}}{\kappa_{g} \pm \kappa_{n} \sqrt{\kappa_{n}^{2}+\kappa_{g}^{2}-1}}\right)
$$

Case (2) occurs when $\tau_{g}=0\left(\theta^{\prime}=\tau\right)$, and $1-$ $\kappa_{g} \cos \vartheta-\kappa_{n} \sin \vartheta \neq 0$. Thus, from Eqs. (10), and (11) it can be found that $g_{12}=h_{12}=0$. Thus, the $\vartheta$-and $s$ curves of $M$ are lines of curvature. Namely, the Darboux frame turn out to the so called Bishop frame or rotation minimizing frame (RMF) [4, 6].

Surfaces whose parametric curves are lines of curvature have several applications in geometric design. In the case of sweeping surfaces, one has to compute the offset surfaces $\mathbf{Q}_{f}(s, \vartheta)=\mathbf{Q}(s, \vartheta)+f \mathbf{N}(s, \vartheta)$ of a given surface $\mathbf{Q}(s, \vartheta)$ at a certain distance $f$. In consequence of this equation, the offsetting operation for sweeping surface can be reduced to the offsetting of planar profile curve, which is much easier to deal with. Hence, we can state the following proposition:

Proposition 3.4. Consider a sweeping surface $M$ represented by Eq. (7). Let $\mathbf{x}_{f}(\vartheta)$ be the planar offset of the profile $\mathbf{x}(\vartheta)$ at distance $f$. Then the offset surface $\mathbf{Q}_{f}(s, \vartheta)$ is again a sweeping surface, generated by the spine curve $\alpha(s)$ and profile curve $\mathbf{x}_{f}(\vartheta)$.

Through the reminder of this work we will study sweeping surfaces characterized by $\tau_{g}=0$, and $1-\kappa_{g} \cos \vartheta-\kappa_{n} \sin \vartheta \neq 0$. Therefore, the value of one principal curvature is

$$
\chi_{1}:=\left\|\frac{d \mathbf{x}}{d \vartheta} \times \frac{d^{2} \mathbf{x}}{d \vartheta^{2}}\right\|\left\|\frac{d \mathbf{x}}{d \vartheta}\right\|^{-3}=1 .
$$


The other principal curvature is easy to get

$$
\chi_{2}=\frac{K(s, \vartheta)}{\chi_{1}}=-\frac{\kappa_{g} \cos \vartheta+\kappa_{n} \sin \vartheta}{1-\kappa_{g} \cos \vartheta-\kappa_{n} \sin \vartheta} .
$$

To analyze the shape of $\mathbf{Q}(\vartheta, s)$ we investigate the distribution of the Gaussian curvature $K(s, \vartheta)$ in the following: The curvature of the isoparametric $s$-curves (u-constant) is

$$
\chi:=\frac{\left\|\mathbf{Q}_{s} \times \mathbf{Q}_{s s}\right\|}{\left\|\mathbf{Q}_{s}\right\|^{3}}=\frac{\kappa}{1-\kappa_{g} \cos \vartheta-\kappa_{n} \sin \vartheta} .
$$

Furthermore, from Eqs (2), and (9) we see that

$$
\mathbf{N}(s, \vartheta)=\cos \varphi \mathbf{n}+\sin \varphi \mathbf{b}, \text { with } \varphi=\vartheta-\theta .
$$

Here $\varphi$ is the angle from $\mathbf{n}$ to $\mathbf{N}$ in the orientation of the tangent plane $T(M)$. On the other hand, the principal curvature $\chi_{2}$ is related to the curvature $\chi(s, \vartheta)$ via Meusnier's Theorem [1]:

$$
\chi_{2}=\chi(s, \vartheta) \cos \varphi .
$$

Therefore, the Gaussian curvature $K(s, \vartheta)$ can be rewritten as

$$
K(s, \vartheta)=\chi(s, \vartheta) \cos \varphi .
$$

We now need to find curves at $M$ that are generated using parabolic points, which means points with vanishing Gaussian curvature. These curves separate elliptic ( $K>0$, locally convex) and hyperbolic $(K<0$, hence non-convex) parts of the surface. In computer Aided Geometric Design, conditions that guarantee the convexity of a surface are required in various applications (such as manufacturing of sculptured surfaces, or layered manufacturing). In the case of the sweeping surface $M$, however, the convexity can be controlled with the help of the differential geometric properties as:

$$
K(s, \vartheta)=0 \Leftrightarrow \chi(s, \vartheta) \cos \varphi=0 .
$$

It can be seen that there is two possible cases producing parabolic points:

Case (1) occurs if $\chi(s, \vartheta)=0$. Using Eq. (23) leads to that in case $\kappa(s)=0$, therefore $\chi=0$. In other words, the spine curve $\alpha=\alpha(s)$ is degenerate into the straight line. Therefore, the inflection or the flat point of this spine curve generates the parabolic curve $\vartheta=$ const. at the sweeping surface.

Case (2) occurs if $\varphi=\pi / 2$. This means that in case $N(s, \vartheta) \| \mathbf{b}$, thus $\cos \varphi=0$. Therefore this curve $\alpha(s)$ is not just the line of curvature but it is asymptotic curve also of the sweeping surface.
Hence, for the existing of parabolic points, the condition

$$
\cos \varphi=0 \Leftrightarrow \vartheta-\theta=\frac{\pi}{2},
$$

is satisfied. In fact we give the next corollary:

Corollary 3.2. Consider the sweeping surface $M$ with spine and profile curves have non-vanishing curvatures everywhere. If the normal $\mathbf{N}(s, \vartheta)$ is never parallel to the principal normal $\mathbf{n}(s)$ of the spine curve $\alpha(s)$, therefore $M$ has no parabolic points.

According to Proposition 3.3, with attention to $\tau_{g}=0$, Eqs. (7), and (28) the expression of the two parabolic curves is

$$
\left.\begin{array}{c}
\Gamma_{1}(s)=\alpha(s)-\frac{\kappa_{n}+\kappa_{g} \sqrt{\kappa_{n}^{2}+\kappa_{g}^{2}-1}}{\kappa_{n}^{2}+\kappa_{g}^{2}} \mathbf{e}_{2} \\
+\frac{\kappa_{g}+\kappa_{n} \sqrt{\kappa_{n}^{2}+\kappa_{g}^{2}-1}}{\kappa_{n}^{2}+\kappa_{g}^{2}} \mathbf{e}_{3}, \\
\Gamma_{2}(s)=\alpha(s)-\frac{\kappa_{n}-\kappa_{g} \sqrt{\kappa_{n}^{2}+\kappa_{g}^{2}-1}}{\kappa_{n}^{2}+\kappa_{g}^{2}} \mathbf{e}_{2} \\
+\frac{\kappa_{g}-\kappa_{n} \sqrt{\kappa_{n}^{2}+\kappa_{g}^{2}-1}}{\kappa_{n}^{2}+\kappa_{g}^{2}} \mathbf{e}_{3} .
\end{array}\right\}
$$

Corollary 3.3. Suppose $M$ is the sweeping surface with spine and profile curves have nonvanishing curvatures everywhere. Therefore $M$ has two parabolic curves if and only if the spine curve $\alpha(s)$ is the asymptotic curve.

\subsection{Developable surfaces}

Developable surfaces can be briefly defined as special cases of the ruled surfaces. Such surfaces are used in different things, for example, in the manufacture of automobile body parts, airplane wings, and ship hulls [15-20]. As a result, we analyze the case, that the characteristic circle $\mathbf{x}(\vartheta)$ degenerates into the straight line, i.e. $\mathbf{x}(\vartheta)=(0, \vartheta, \vartheta)$, thus generating a developable ruled surface

$$
S: \mathbf{P}(s, \vartheta)=\alpha(s)+\vartheta \mathbf{e}_{3}(s), \vartheta \in R .
$$

Similarly, from Eq. (7), the developable surface is written as

$$
S^{\perp}: \mathbf{P}^{\perp}(s, \vartheta)=\alpha(s)+\vartheta \mathbf{e}_{2}(s), \vartheta \in R .
$$

It is possible to show $\mathbf{P}(s, 0)=\alpha(s)$ (resp. $\left.\mathbf{P}^{\perp}(s, 0)=\alpha(s)\right), 0 \leq s \leq L$, such that , the surface $S$ (resp. $S^{\perp}$ ) interpolate the curve $\alpha(s)$. Furthermore, since

$$
\mathbf{P}_{s} \times \mathbf{P}_{\vartheta}:=-\left(1-\vartheta \kappa_{n}\right) \mathbf{e}_{2}(s),
$$


then $S^{\perp}$ is the normal developable surface of $S$ along $\alpha(s)$. Therefore, the surface $S$ (resp. $S^{\perp}$ ) interpolates the curve $\alpha(s)$, and $\alpha(s)$ is the line of curvature of $S$ (resp. $S^{\perp}$ ).

Proposition 3.5. (Existence and uniqueness). Considering previous notations there is the unique $\mathbf{P}(s, \vartheta)$ developable ruled surface represented by Eq. (30).

Proof. For the existence, we have the $\mathbf{P}(s, \vartheta)$ developable ruled surface represented by Eq. (30). Moreover, since $\mathbf{P}(s, \vartheta)$ is the ruled surface, we assume that

$$
\left.\begin{array}{c}
S: \mathbf{P}(s, \vartheta)=\alpha(s)+\vartheta \zeta(s), \vartheta \in R, \\
\text { with }\left(\kappa_{g}, \kappa_{n}\right) \neq(0,0), \\
\zeta(s)=\zeta_{1}(s) \mathbf{e}_{1}+\zeta_{2}(s) \mathbf{e}_{2}+\zeta_{3}(s) \mathbf{e}_{3}, \\
\|\zeta(s)\|^{2}=\zeta_{1}^{2}+\zeta_{2}^{2}+\zeta_{3}^{2}=1, \zeta^{\prime}(s) \neq \mathbf{0} .
\end{array}\right\}
$$

Direct result from Eqs. (38) shows that $S$ is developable if and only if

$$
\begin{gathered}
\operatorname{det}\left(\alpha^{\prime}, \zeta, \zeta^{\prime}\right)=0 \Leftrightarrow \\
-\zeta_{3} \zeta_{2}^{\prime}+\zeta_{2} \zeta_{3}^{\prime}-\zeta_{1}\left(\zeta_{3} \kappa_{g}-\zeta_{2} \kappa_{n}\right)=0 .
\end{gathered}
$$

Further, since $S$ is a developable surface along $\alpha=\alpha(s)$, we have

$$
\left(\mathbf{P}_{s} \times \mathbf{P}_{\vartheta}\right)(s, \vartheta)=\psi(s, \vartheta) \mathbf{e}_{2},
$$

where $\psi=\psi(s, \vartheta)$ is the differentiable function. In addition, the normal vector $\mathbf{P}_{s} \times \mathbf{P}_{\vartheta}$ at the point $(s, 0)$ is

$$
\left(\mathbf{P}_{s} \times \mathbf{P}_{\vartheta}\right)(s, 0)=-\zeta_{3} \mathbf{e}_{2}+\zeta_{2} \mathbf{e}_{3} .
$$

Thus, from Eqs. (32), and (36), one finds that:

$$
\zeta_{3}=0, a n d \zeta_{2}=\psi(s, 0),
$$

which follows from Eq. (34) that $\zeta_{1}\left(\zeta_{2} \kappa_{n}\right)=0$. In case $(s, 0)$ is the regular point (which means, $\psi(s, 0) \neq 0)$, therefore $\zeta_{1}=0$, and $\zeta_{2}(s) \neq 0$. Therefore, we obtain $\zeta(s)=\mathbf{e}_{2}$.

Proposition 3.6. Suppose $M$ is a sweeping surface defined using Eq. (7). As a result we can have:

(1) The developable surfaces $S$ and $S^{\perp}$ intersect along $\alpha(s)$ at a right angle,

(2) The curve $\alpha(s)$ is the line of curvature on $S$ and $S^{\perp}$.
As an application (such as cylindrical milling or flank milling), among the motion of the Darboux frame, consider the cylindrical cutter be rigidly linked to this frame. Therefore the equation of the family of cylindrical cutters, which is given using the movement of cylindrical cutter through $\alpha(s)$, will be written as:

$$
S_{f}: \overline{\mathbf{P}}(s, \vartheta)=\mathbf{P}(s, \vartheta)+f \mathbf{e}_{2}(s),
$$

where $f$ defines cylindrical cutter radius. This surface is the developable surface offset of the surface $\mathbf{P}(s, \vartheta)$. The equation of $S_{f}$, will be given as

$$
S_{f}: \overline{\mathbf{P}}(s, \vartheta)=\alpha(s)+\vartheta \mathbf{e}_{3}(s)+f \mathbf{e}_{2}(s) .
$$

The normal vector of cylindrical cutter is presented as

$$
\frac{\overline{\mathbf{P}}_{s} \times \overline{\mathbf{P}}_{\vartheta}}{\left\|\overline{\mathbf{P}}_{s} \times \overline{\mathbf{P}}_{\vartheta}\right\|}=\mathbf{e}_{2}(s) .
$$

Also, from Eq. (38), we have

$$
S: \mathbf{P}(s, \vartheta)=\overline{\mathbf{P}}(s, \vartheta)-f \mathbf{e}_{2}(s) .
$$

The derivative of Eq. (41) respecting to $s$ is introduced as

$$
\overline{\mathbf{P}}_{s}(s, \vartheta)=\mathbf{P}_{s}(s, \vartheta)-(f \omega) \times \mathbf{e}_{2} .
$$

From Eq. (42) it can be seen that the vector $\overline{\mathbf{P}}_{s}(s, \vartheta)$ is perpendicular to the normal vector $\mathbf{e}_{2}$. Further, the vector $\mathbf{e}_{2}$ is perpendicular to the tool axis vector $\mathbf{e}_{1}(s)$. Consequently, the envelope surface of the cylindrical cutter and the developable surface $\mathbf{P}(s, \vartheta)$ have the common normal vector and the length between the two surfaces is cylindrical cutter radius $f$. Hence, this is will be given as coming:

Proposition 3.7. Consider a developable surface $S$ parametrized by Eq. (30). Let $S_{f}$ be the envelope surface of cylindrical cutter at distance $f$. Therefore the two surfaces $S$ and $S_{f}$ are offset developable surfaces.

\subsection{Application}

As an application of our main results, an representative example is given. It is also used to be sure of the previous formulae.

Example 1. Given the base surface as follows: $\mathbf{X}(s, v)=\left(\cos s-\frac{v}{\sqrt{2}} \cos s, \sin s-\frac{v}{\sqrt{2}} \sin s, \frac{v}{\sqrt{2}}\right)$, where $I \subseteq R$, and $v \in R$. According to

$$
\mathbf{e}_{3}(s, v)=\frac{\mathbf{X}_{s} \times \mathbf{X}_{v}}{\left\|\mathbf{X}_{s} \times \mathbf{X}_{v}\right\|} \text {, and } \mathbf{e}_{3}(s, 0)=\mathbf{e}_{3}(s),
$$


we get

$$
\mathbf{e}_{3}(s)=\left(\frac{1}{\sqrt{2}} \cos s, \frac{1}{\sqrt{2}} \sin s, \frac{1}{\sqrt{2}}\right) .
$$

Moreover, we have

$$
\mathbf{e}_{1}(s)=(-\sin s, \cos s, 0) .
$$

Since $\mathbf{e}_{2}(s)=\mathbf{e}_{3}(s) \times \mathbf{e}_{1}(s)$,

$$
\mathbf{e}_{2}(s)=\left(-\frac{1}{\sqrt{2}} \cos s,-\frac{1}{\sqrt{2}} \sin s, \frac{1}{\sqrt{2}}\right) .
$$

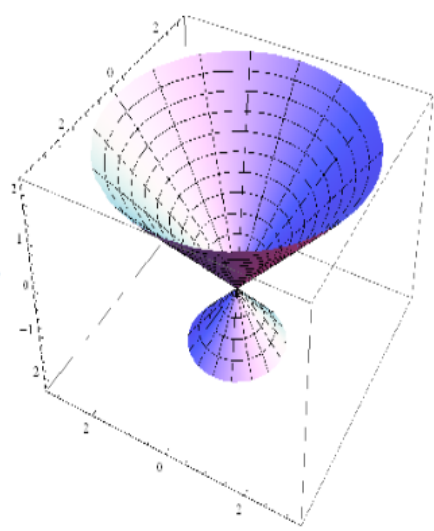

Figure 2: $S$

Also, we can calculate

$$
\kappa_{g}=\frac{1}{\sqrt{2}}, \kappa_{n}=\frac{-1}{\sqrt{2}} \text {, and } \tau_{g}=0 \text {. }
$$

Then $\theta(s)=\theta_{0}$ is a constant. Therefor, the sweeping surface can be given as

$$
\begin{gathered}
M: \mathbf{Q}(s, \vartheta)=(\cos s, \\
\sin s, 0)+\cos \vartheta \mathbf{e}_{2}(s) \\
+\sin \vartheta \mathbf{e}_{3}(s) .
\end{gathered}
$$

The graphs of the sweeping surface and the developable surfaces are shown in Figs. 1, 2, and 3 . Note that the developable surfaces $P$ and $P^{\perp}$ intersect along $\alpha(s)$ at a right angle as seen in the Fig. 4.

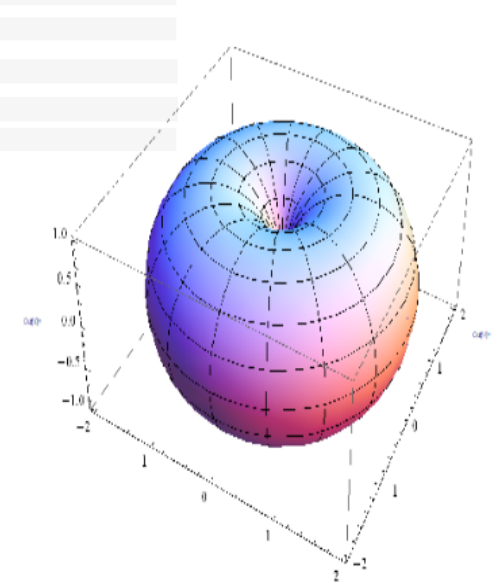

Figure 1: $M$ 
frames. The paper further investigated the problem of requiring the surfaces that are being both sweeping surfaces and developable surfaces. We hope those results will be used more for surfaces in geometric modeling, garment-manufacture industry, and the manufacturing of products. The methodology used here can be applied in different spaces such as Lorentz-Minkowski space, isotropic space, and etc.

\section{Acknowledgements}

This research was funded by the Deanship of Scientific Research at Princess Nourah bint Abdulrahman University through the Fast-track Research Funding Program. The authors gratefully acknowledge the reviewers whose comments assisted in improving this work.

\section{References:}

[1] MP. Do Carmo. Differential Geometry of Curves and Surfaces, Prentice Hall, Englewood Cliffs, NJ, 1976.

[2] B. Yıldız, K. Arslan, H. Yıldız, C. Ozgür. A geometric description of the ascending colon of some domestic animals. Ann Anat, vol.183, No.6, 2001, pp 555-557.

[3] PA. Blaga. On tubular surfaces in computer graphics. Stud Univ Babeş-Bolyai Inform, 2005, pp 81-90.

[4] W. Wang, B. Jüttler, D. Zheng \& Y. Liu. Computation of rotating minimizing frames. ACM Transactions on Graphics,vol. 27, No.1, 2008, pp 1-18.

[5] MK. Karacan, Y. Yaylı. On the geodesics of tubular surfaces in Minkowski 3-space. Bull Malays Math Sci Soc; vo;.31, No.1, 2008, pp $1-10$.

[6] RT. Farouki \& T. Sakkalis. Rational rotationminimizing frames on polynomial space curves of arbitrary degree,. J Symb Comput,vol. 45, No.8, 2010, pp 844-856.

[7] Karacan MK, Yoon DW, Tunçer Y. Weingarten and linear Weingarten type tubular surfaces in E3 . Math Prob in Eng 2011, doi. no: $10.1155 / 2011 / 191849$.

[8] F. Doğan. Generalized Canal Surfaces. PhD, Ankara University Graduate School, Ankara, Turkey, 2012.

[9] S. Aslan, Y. Yayl. Canal surfaces with quaternions. Adv Appl Clifford Algebras; vol. 26No.1, 2015, pp 31-38.
[10] I Gok. Quaternionic approach of canal surfaces constructed by some new ideas. $A d v$ in Appl Clifford Algebras, vol.27, No.2, 2017, pp $1-16$.

[11] RA. Abdel-Baky \& Y. Ynlütürk. On the curvatures of spacelike circular surface, Kuwait Journal of Science, vol. 43, Vol.3, 2016.

[12] H. Shichang, W. Zhigang \& T. Xiaoqing. Tubular surfaces of center curves on spacelike surfaces in Lorentz-Minkowski 3-space, Math Meth Appl Sci, 2018, pp1-31.

[13] RA. Abdel-Baky, N. Alluhaibi, A. Ali \& F. Mofarreh. A study on timelike circular surfaces in Minkowski 3-Space. Int. J. of Geometric Methods in Modern Physics, vol. 17, No. 06, 2020.

[14] RA. Abdel-Baky. Developable surfaces through sweeping surfaces, Bulletin of the Iranian Mathematical Society, Vol. 45, no. 4, 2019, pp 951-963.

[15] H.Y. Zhao, and G.J. Wang. A new method for designing a developable surface utilizing the surface pencil through a given curve. Progress in Nature Science, vol. 18, 2008, pp $105-10$.

[16] C.Y. Li, R.H. Wang, and C.G. Zhu. An approach for designing a developable surface through a given line of curvature, ComputerAided Design, vol. 45, 2013, pp 621-627.

[17] O.G Yıldız, S.O. Karakus, and H.H. Hacisalihoglu. On the determination of a developable spherical orthotomic ruled surface, Bull. Math. Sci. vol. 5, 2015, pp 137-146.

[18] R.A. Abdel-Baky \& Y. Unlütürk. Normal developable surfaces of a surface along a space curve, J. Indones. Math. Soc. Vol. 26, No. 03,2010, pp. 319-333.

[19] R.A. Abdel-Baky \& F. Mofarreh. On the determination of ruled and developable surfaces in Euclidean 3-space, WSEAS Transactions on Mathematics DOI: 10.37394/23206.19.60, (2020).

[20] R.A. Abdel-Baky \& Y. Unlütürk. A new construction of timelike ruled surfaces with constant Disteli-axis, Honam Math. Journal,vol. 42, No. 3,2020, pp. 551-568.

Follow: www.wseas.org/multimedia/contributorrole-instruction.pdf 


\section{Creative Commons Attribution License 4.0} (Attribution 4.0 International, CC BY 4.0)

This article is published under the terms of the Creative Commons Attribution License 4.0

https://creativecommons.org/licenses/by/4.0/deed.en_US 